\title{
Editorial
}

\section{New tuberculosis vaccine to support tuberculosis elimination}

\author{
Fransisca Chondro \\ Department of Physiology Faculty of Medicine, Trisakti University
}

The Sustainable Development Goals have prioritized ending the epidemic of tuberculosis by 2030. ${ }^{(1)}$ Around one-third of the world population is infected with Mycobacterium tuberculosis (MTBC), but is asymptomatic, a conditon known as latent TB. ${ }^{(2)}$ According to the global tuberculosis report, in 2017 there were $5-15 \%$ of 1.7 billion persons infected with MTBC, who will develop TB in their lives. ${ }^{(1)}$ Around $10 \%$ of latent TB will develop into active TB disease, with a higher risk in patients with immunodeficiencies such as HIV, undernutrition, diabetes mellitus, smoking, and habitual alcohol consumption. As a result there are annually an estimated 8.8 million new TB cases, with a TB mortality of 1.1 million and deaths from TB and HIV coinfection of 350 thousand cases. ${ }^{(1,3)}$ The World Health Organization has planned a number of strategies for TB elimination in the year 2030, such as developing rapid and accurate diagnostic tests, new regimens for the treatment of drug-susceptible or drugresistant TB, prevention of transmission of $M$. tuberculosis through infection control, new vaccine candidates to prevent the development of TB, and to help improve the outcomes of treatment for TB disease. ${ }^{(1)}$

Based on the estimated incidence of TB, the WHO published a list of countries with the highest numbers of cases of TB, TB-HIV, and multi-drug resistant TB (MDR-TB). Indonesia is included in all categories; in other words, Indonesia belongs to the 20 countries with the highest incidence of TB, TB-HIV, and MDRTB. ${ }^{(1)}$
Natural infection induces the production of antibodies against Mycobacterium tuberculosis (MTBC). Studies show that $90 \%$ of TB patients have a higher serum immunoglobulin titer against MTBC at the onset of the clinical symptoms of the disease. However, there are a wide variety of antigens that produce the target antibody. One of the studies show that among 10 culture results of MTBC protein filtrates, there was not one antigen that is found in the serum of patients with active MTBC..$^{(1)}$

In the last few decades, several DNA fingerprinting techniques have been used to differentiate between a number of MTBC strains. Restriction fragment length polymorphism (RFLP) is based on the number of DNA copies and the genomic location of the IS6110 insertion sequence. This technique is the gold standard for examining the actual transmission stage of MTBC bacteria in the patient's body, for differentiating between relapse and reinfection in the patient, and for detecting cross-contamination in the laboratory. IS6110 is an insertion sequence of 1361 base pairs, with inverted repeats of 28 base pairs at each end. Restriction fragment length polymorphism analysis of IS6110 depends on the digestion of DNA genetic material by endonucleases followed by electrophoretic separation of the DNA fragments, which are then transferred to a nylon or nitrocellulose membrane. The fragments on the membrane are then subjected to Southern blot hybridization with a labeled DNA probe, which will adhere to the 
IS6110-specific DNA fragment. Two limitations of this technique are the difficulty of obtaining comparable results in different laboratories and the need for DNA of good quality in large amounts. Therefore, several PCR-based methods have been developed to assist in the identification of MTBC strains using relatively small amounts of DNA. In this category belong spoligotyping and mycobacterial interspersed repeat units (MIRUs) typing, both of which are considered the new gold standards in molecular epidemiological studies of MTBC..$^{(3,4)}$

To date many studies seek to evaluate the effect of MTBC strain variations on virulence and immunogenicity. Virulence of TB may be influenced by the ability of the bacteria to survive after facing the host immune response, to damage the lungs, to survive aerosolization outside the host, and to be transmitted to healthy persons. Studies in humans and experimental animals have shown that TNF-alpha, gamma interferon, IL-12 and IL17 are important mediators for the formation of the immune response of the patients against MTBC. ${ }^{(4)}$ Other mediators such as IL-10 are also thought to play a role in limiting the development of MTBC at the initial stages of the immune response. Previously it was concluded that the more severe the inflammatory response, the more severe the damage to the lungs, increasing the efficiency of MTBC transmission. However, several studies found that the increase in MTBC virulence was associated with a decreased and/ or delayed inflammmatory response, possibly as a result of considerable bacterial proliferation in the early stages of infection and leading to increased MTBC virulence in the final stages. ${ }^{(5,6)}$

The Bacille Calmette-Guérin (BCG) vaccine, which was developed almost 100 years ago and has been shown to prevent severe forms of TB in children, is still widely used. However, there is currently no vaccine that is effective in preventing TB disease in adults, either before or after exposure to TB infection.

The BCG vaccine is considered to be less effective, since it protects only against $\mathrm{TB}$ infection in childhood, but does not influence the development of TB infection or reinfection in adults. In addition, the vaccine shows variable efficacy due to differences in the BCG strain used. Therefore, there are currently several initiatives, such as the development of a better vaccine than the present $B C G$ vaccine, the administration of a booster with a second dose of BCG or the administration of a BCG vaccine containing a different strain.

To date there are 12 types of vaccine under study. For phase I studies, there are 3 vaccines, i.e. i). Ad5 Ag85A - adenovirus serotype 5 expressing Ag85A. This vaccine will be administered by the intramuscular route after it has been demonstrated to be safe, is tolerated, and increases the patient's immune level with a higher immunogenicity if administered to patients who have previously received the $\mathrm{BCG}$ vaccine;ii). ChAdOx185A - MVA85A. This vaccine is a combination of a simian adenovirus and a recombinant pox virus, both of which express the $85 \mathrm{~A}$ antigen. The ChAdOx185A vaccine is for intramuscular administration and has been tested in a phase I trial. At present the phase I trial for its administration in aerosol form is ongoing;iii) MTBVAC. This is a live MTBC strain attenuated through deletions in the genes phoP and fadD26. The main target population of this vaccine are the neonates (for replacing the current $B C G$ vaccine). In addition, this vaccine may also be administered to adolescents and adults as repeat vaccine (booster).

For phase II and III studies, there are 9 vaccines under development: i). DAR-901 booster is a vaccine that consists of nontuberculous mycobacteria, in whole-cell and heat-inactivated form;ii). H4:IC31 is a booster vaccine containing a fusion protein of $\mathrm{Ag} 85 \mathrm{~B}$ and TB10.4 with IC 31 adjuvant. This vaccine is under trial to evaluate its efficacy in preventing infection in adolescents at high risk for MTBC infection, and has been tested for administration to infants;iii). H56:IC31 is a vaccine comprising a combination of three MTBC antigens (Ag85B, ESAT-6, and Rv2660c) with IC31 adjuvant. This vaccine has been tested, proven to be safe and 
possesses substantial immunogenicity;iv). ID93

+ GLA-SE is a vaccine consisting of 4 MTBC antigens associated with virulence ( $\mathrm{Rv} 2608$, Rv3619 and Rv3620) or latency (Rv1813), and the adjuvant GLA-SE;v). M72/AS01E is a subunit vaccine containing 2 MTBC antigens (32A and 39A) with an adjuvant (AS01E);vii). RUTI ${ }^{\circledR}$ is a therapeutic vaccine containing MTBC cell-wall fragments for co-administration with short-term antibiotics. The main targets of this vaccine are patients with MDR-TB;vii). TB/ FLU-04L is a mucosal-vectored vaccine containing influenza virus with a replication deficiency that has been attenuated and expresses the Ag85A and ESAT-6 antigens. This vaccine is intended as prophylactic boost vaccine for infants, adolescents, and adults; viii). Vaccae ${ }^{\mathrm{TM}}$ is an immunotherapeutic agent intended to shorten the therapeutic period in patients with MTBC infections that are still susceptible to the basic MTBC therapy; and ix). VPM1002 is a recombinant vaccine containing live MTBC bacteria. This vaccine is under trial to evaluate its safety and immunogenicity in neonates exposed to the HIV virus and those unexposed to HIV.(1)

It is hoped that with the increased understanding and knowledge about the host and the MTBC bacteria, the goal of developing a suitable vaccine may be achieved, so that TB may be expected to be eliminated in the year 2030 .

\section{REFERENCES}

1. World Health Organiztaion. Global tuberculosis report 2017. Geneva: World Health Organization; 2017.

2. Achkar JM, Casadevall A. Antibody-mediated immunity against tuberculosis: implications for vaccine development. Cell Host Microbe 2013; 13:250-62. doi: http://dx.doi.org/10.1016/j.chom. 2013.02.009.

3. Fogel N. Tuberculosis: a disease without boundaries. Tuberculosis 2015;95:527-31. doi: http://dx.doi.org/10.1016/j.tube.2015.05.017.

4. Coscolla M, Gagneux S. Consequences of genomic diversity in Mycobacterium tuberculosis. Seminars Immunology 2014;26:43144. doi: http://dx.doi.org/10.1016/j.smim.2014.09. 0121044-5323.

5. Mbugi EV, Katale BZ, Siame KK, et al. Genetic diversity of Mycobacterium tuberculosis isolated from tuberculosis patients in the Serengeti ecosystem in Tanzania. Tuberculosis. 2015;95: 170-8. doi: http://dx.doi.org/10.1016/j.tube.2014. 11.006 .

6. Cadmus S, Hill V, van Soolingen D, et al. Spoligotype profile of Mycobacterium tuberculosis complex strains from HIV-positive and -negative patients in Nigeria: a comparative analysis. J Clin Microbiol 2011;49:220-6. doi: 10.1128/JCM.01241-10. 